
WITH TUMOR-LIKE PRESENTATION

C. Salvarani ${ }^{1}$, R.D. Brown ${ }^{2}$, T.J. Christianson ${ }^{2}$,C. Giannini ${ }^{2}$,J. Huston $\mathrm{II}^{2}, \mathrm{G}$. G. Hunder ${ }^{2}$. Azienda USL-IRCCS di Reggio Emilia e Università di Modena e Reggio Emilia, Reggio Emilia, Italy; ${ }^{2}$ Mayo Clinic, Rochester, USA

Background: Primary central nervous system vasculitis (PCNSV) rarely presents as a tumor-like mass lesion (ML).

Objectives: The aim of this study was to determine the frequency of ML in a large cohort of patients with PCNSV and compare the presenting clinical, laboratory, and imaging features in those with $\mathrm{ML}$ to those without.

Methods: We retrospectively studied a cohort of 191 consecutive patients with PCNSV who were seen at the Mayo Clinic, Rochester, MN over a 35 year period. The diagnosis of PCNSV was based on brain/spinal cord biopsy or cerebral angiography. We compared PCNSV patients with tumor-like presentation to those without.

Results: 13/191 (6.8\%) patients had tumor-like presentation. In all 13 patients PCNSV diagnosis was established by cerebral biopsy (stereotactic in 10, openwedge in 3). 4 patients had cerebral angiography, and vasculitis was suggested in one patient. A granulomatous inflammatory histologic pattern was found in 11 biopsies, accompanied by vascular deposits of $\beta$-amyloid peptide in 7 . In the other 2 biopsies a lymphocytic vasculitis was observed. The 13 patients with tumor-like presentation were compared with the 178 patients without. The patients with ML were more frequently males $(77 \%$ vs $44 \%, p=0.04)$, were less likely to present with transient ischaemic attacks (TIA) ( 0 vs $27.5 \%, p=0.023$ ) and more likely to present with seizures $(46 \%$ vs $17 \%, p=0.022)$ at presentation. No significant differences in the CSF findings and ESR levels (normal in $100 \%$ and $82 \%$ of the patients, respectively) at diagnosis were observed in the two groups. Intracerebral gadolinium-enhancing lesions were more frequently observed in patients with $\mathrm{ML}$ ( $46 \%$ vs $21 \%, p=0.07$ ), while meningeal gadolinium-enhancing lesions were equally observed ( $31 \%$ vs $20 \%, p=0.48)$. The frequencies of PCNSV recurrence (38\% vs $29 \%$ ), patients not requiring therapy at last follow-up (15\% vs $25 \%$ ), response to therapy (100\% vs $74 \%$ ), and poor outcomes (modified Rankin disability score $\geq 4$ ) at last followup ( $8 \%$ vs $26 \%$ ) were not significantly different in the two groups. No differences in survival were observed between the 2 groups $(p=0.57)$. Considering all 191 patients, univariate Cox proportional hazards modelling showed an increased mortality rate in those with increasing age (hazard ratio, HR, 1.4), cerebral infarction on initial MRI (HR 2.95), and angiographic large vessel involvement (HR 3.2), while mortality rate was lower in those with gadoliniumenhancing lesions on MRI (HR=0.3).

Conclusions: Tumor-like presentation represents a subset of PCNSV characterised by vascular deposits of $\beta$-amyloid at biopsy, seizures at presentation, and gadolinium-enhancing cerebral lesions on MRI. As in PCNSV without ML, treatment response and prognosis was favourable in most patients.

Disclosure of Interest: None declared

DOI: 10.1136/annrheumdis-2018-eular.5036

\section{FRI0480 TOCILIZUMAB MONO-THERAPY FOR POLYMYALGIA RHEUMATICA RESULTS OF 104-WEEK TREATMENT OF A PROSPECTIVE, SINGLE-CENTRE, OPEN TRIAL}

K. Amano, K. Chino, Y. Okada, A. Shibata, S. Saito, T. Kurasawa, A. Okuyama, H. Takei, R. Sakai, T. Kondo. Rheumatology and Clinical Immunology, Saitama Medical Center, Saitama Medical University, Kawagoe, Japan

Background: Polymyalgia rheumatic (PMR) is a chronic inflammatory rheumatic disease in the elderly people. Glucocorticoid (GC) is still definitely a mainstay for the treatment of PMR, but long-term GC therapy is a major risk factor of osteoporotic fractures, diabetes, hyperlipidemia, cardio-cerebral vascular events, glaucoma, etc. So GC-free treatment strategies for PMR are awaited for some PMR patients with these comorbidities. IL-6 is involved in the pathogenesis of PMR and several case reports have already shown the efficacy of tocilizumab (TCZ), anti$\mathrm{IL}-6$ receptor antibody, in PMR patients ${ }^{1,2)}$ and there is a report of TCZ mono-therapy as the first-line therapy in $\mathrm{PMR}^{3}$.

Objectives: To assess the efficacy and safety of TCZ mono-therapy for PMR Methods: Thirteen PMR patients (male 3, female 10) who had been diagnosed by 2012 ACR/EULAR classification criteria from Jan 2013 to Feb 2015 were enrolled in our single-centre, prospective study (IRB application No. 638, UMIN No. 000008812) after obtaining the written informed consent. TCZ (8 mg/kg) was administered biweekly for the first 8 weeks ( 5 infusions) and every 4 weeks thereafter for 40 weeks (total 15 infusions). ESR, CRP, patient's global health assessed by visual analogue scale (VAS), PMR activity score ${ }^{4)}$ were evaluated every 4 week prospectively. Primary endpoint was remission rate at week 52 . Remission was defined as PMR activity score less than $1.5^{4)}$. Patients were followed up for another one year without any treatment and flare rate was assessed at week 104.

Results: Baseline patients' characteristics revealed various kinds of comorbidities in 11 patients; hypertension in 7 , hyperlipidemia in 5 , diabetes mellitus in 3 , osteoporotic vertebral fractures in 2, history of angina pectoris in 1, history of brain infarction in 1, history of hematemesis due to NSAID ulcer in 1 and glaucoma in one patient. Nine patients could complete this 104 week trial and all 9 patients could achieve remission at week 52 and 8 of 9 patients fulfilled remission criteria at week 104. Two patients discontinued TCZ because of no response at week 6 (No.1) and week 16 (No. 8) respectively. One patient (No.2), who were in clinica remission of PMR, dropped out from this study due to pemphigoid at week 50 and received GC therapy. Patient No. 12 abandoned TCZ at week 12 because of lung infiltrates although she was treated successfully with TCZ mono-therapy, and she had been in remission without any treatment until week 104. The other 3 patients could obtain remission with GC therapy at week 52 . There were no serious adverse events during 104 week treatment period.

Conclusions: TCZ mono-therapy was effective in most (9 out of 13) PMR patients although response was not so rapid as compared to GC. TCZ mono-therapy may be a good alternative therapy instead of GC for elderly patients with various comorbidities.

\section{REFERENCES:}

[1] Unizony S, et al.: Arthritis Care Res 2012;64(11):1720-1729.

[2] Macchioni $P$, et al.: Semin Arthritis Rheum 2013;43(1):113-118.

[3] Devauchelle-Pensec V, et al.: Ann Rheum Dis 2016;75(8):1506-1510.

[4] Leeb BF, et al.: Arthritis Rheum 2007;57(5):810-815.

Disclosure of Interest: K. Amano Grant/research support from: Chugai Pharmaceutical Co. Ltd., Speakers bureau: Chugai, Daiichi-Sankyo, Pfizer Japan, Tanabe-Mitsubishi, K. Chino: None declared, Y. Okada: None declared, A. Shibata: None declared, S. Saito: None declared, T. Kurasawa: None declared, A Okuyama: None declared, H. Takei: None declared, R. Sakai: None declared, T. Kondo: None declared

DOI: 10.1136/annrheumdis-2018-eular.3605

\section{FRI0481 GIANT CELL ARTERITIS AND INFLAMMATORY BOWEL DISEASE - IS THERE A CONNEXION? RESULTS FROM A POPULATION-BASED STUDY}

Y. Yavne ${ }^{1}$, S. Tiosano ${ }^{2}$, A. Watad ${ }^{2}$, D. Comaneshter ${ }^{3}$, A.D. Cohen ${ }^{3}$, H. Amital ${ }^{2}$ ${ }^{1}$ Sackler Faculty of Medicine, Tel Aviv University, Tel Aviv, ${ }^{2}$ Department of Medicine ' $B$ ', Zabludowicz Center for Autoimmune Diseases, Sheba Medical Center, Tel Hashomer, ${ }^{3}$ Chief Physician's Office, Clalit Health Services, Tel Aviv, Israel

Background: Giant cell arteritis (GCA) is an autoimmune disorder which primarily affects large vessels, whilst inflammatory bowel disease (IBD), which includes Crohn's disease (CD) and ulcerative colitis (UC), mainly targets the gut. Co-existence of the two maladies has been reported sporadically in the literature ${ }^{1-3}$.

Objectives: The purpose of this study was to assess the authenticity of such an association in a large, cross-sectional study.

Methods: Utilising data derived from the Clalit Health Services' registry, the largest health maintenance organisation in Israel, we compared the proportion of CD and UC in GCA patients with age- and gender-matched controls. Univariate analysis was performed using Chi-square and student $t$-test and a multivariate analysis was performed using a logistic regression model.

Results: The study included 5,657 GCA patients and 28298 age- and gendermatched controls. GCA patients had a significantly increased proportion of both CD and UC in comparison with controls $(0.81 \%$ vs. $0.12 \%$ and $0.69 \%$ vs. $0.2 \%, p$ value $<0.001$, respectively). The strength of the association between GCA and IBD was negatively correlated with the patients' age; thus the association was most robust amongst younger patients aged 18-44 $(O R=13.2$, figure 1$)$. The association between GCA and IBD remained significant when evaluated independently of confounding factors ( $\mathrm{OR}=2.367, p$-value $<0.001$, table 1 ).

Abstract FRI0481 - Table 1. Multivariate logistic regression of covariates associated with IBD

\begin{tabular}{lccc}
\hline Characteristic & OR & Cl & P \\
\hline Age & 0.99 & $0.98-1$ & 0.49 \\
Gender & 1.25 & $0.87-$ & 0.22 \\
(Female) & & 1.84 & \\
BMI & 0.95 & $0.92-$ & $\mathbf{0 . 0 0 7}$ \\
SES: & & 0.98 & \\
Medium vs. & 1.42 & $0.94-2.1$ & 0.1 \\
Low & & & \\
High vs. Low & 1.74 & $1.13-$ & $\mathbf{0 . 0 1 2}$ \\
GCA & & 2.69 & \\
& 2.36 & $1.71-$ & $<.001$ \\
\hline
\end{tabular}

IBD: Inflammatory bowel disease; BMI: Body Mass Index, kg/m2; SES: Socioeconomic status; GCA: Giant Cell Arteritis 